\title{
Design of Power Quality Monitoring System based on LabVIEW
}

\author{
Wang Shenghui, Chao Yong \\ Institute of Electric and Electronics Engineering \\ Changchun University of Technology \\ Changchun, China
}

\author{
Jin Xing \\ Institute of Electric and Electronics Engineering \\ Changchun University of Technology \\ Changchun, China
}

\begin{abstract}
In the paper, it is introduced that the design of a power quality monitoring system based on LabVIEW for factory power quality, with LabVIEW as development platform, composed by some functional modules such as data acquisition, data processing, data storage, and data report, etc. and making an access to Microsoft Access database using LabSQL to achieve data acquisition, processing, storage and analysis.
\end{abstract}

Keywords- Power quality, LabVIEW, LabSQL, Database

\section{INTRODUCTION}

In recent years, a great quantity of electrical loads characterized by non-linearity, impact strength and unbalance at the user side have caused a serious interference and pollution to the quality of power supplied. The usual problems existing in power quality such as harmonic, asymmetry of the three-phase circuit, voltage fluctuation and flicker, etc. are all becoming worse, while, with the development of high-tech. a number of new-type electrical loads which are sensitive to power quality are developing rapidly, requiring high-quality power to be supplied by power system[1-2]. Nowadays, the voltmeter having a simple statistical function is widely used in China to monitor the voltage, while, these voltmeters can only monitor the qualification rate of voltage, and for lacking the functions of statistics and analysis, it is necessary for us human to read and record the data[3-4]. However, with respect to the measurement of harmonic and voltage fluctuation and flicker, the portable measuring instrument is applied to make an onsite measurement, and according to the data measured, a summarization and statistical analysis will be carried out to make an evaluation on the quality level of the power supplied by the power system. In consideration of the problems existing in the current online monitoring instrument, and in line with the state-level standards concerning power quality in China, an online power quality monitoring system based on LabVIEW which is suitable for the power quality monitoring, management and analysis at the workshop level is designed.

\section{GENERAL DESIGN}

Because autobody panels emphasis curved surface whole artistic effects, measurement accuracy is not the first problem considered in the course of the measuring car body, but trying to make the development time shorter [4].
The hardware structure diagram of the power quality monitoring system is as shown in Figure1, and the hardware of the system includes two computers, and NI Company's SCXI 1000 cases, SCXI 1100 modulation cards and NI PCI6040 data acquisition cards. The monitoring environment is a crushing high-pressure chamber of a crushing workshop of a mining plant, and signals of the on-site voltage and current are converted to the signals applicable for the data acquisition card through voltage and current transformers, then, through the signal conditioner, these signals are conditioned to digital signals to be sent to the data acquisition card, then, the data acquisition card will use a mode of interval scan to control the intervals of both the switching channels and the scanning processes respectively through channel clock and scan clock, for the interval between channels is very small and the processes of scan are in a continuous cycle, the three-phase voltage and current signals of each panel point are approximate to those which are sampled simultaneously, meeting the need from the systematic sampling. The system is divided into two working stations, each of which monitors the voltage and current signals of seven panel points. The software of the system is designed with the application of LabVIEW8.2 graphical programming platform, shows the data collected after a processing, and realizes a timing storage and analysis of the said data through making an access to the database using LabSQL, and then, achieves the power quality parameters' functions of automatic acquisition, storage and analysis.

Finally Functions of the system are as below:

To simultaneously monitor and analyze multipath signals with the detection circuit being divided as the mode of threephase and three-wire;

To measure the effective values of the voltage and current, active power, reactive power, power factor, frequency, the unbalance of the three-phase voltage, the fluctuation ratio of the three-phase voltage, the total harmonic distortion, odd harmonic distortion, even harmonic distortion, and the amplitude value of harmonic at 50th fundamental wave of the three-phase voltage and current;

To show the real-time data of power quality parameters such as voltage, current and harmonic, etc. and the running status of the instrument every $3 \mathrm{~s}$;

To store the measured values into database according to the setting storage interval;

To analyze the historical data and show the status of power quality in a certain period of time as well as the 
maximum value, minimum value, average value, and the time for the maximum value and the minimum value;

To make a report statistics on power quality .

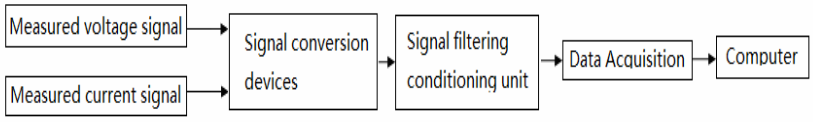

Figure 1. The hardware structure diagram

\section{THE REALIZATION OF LABVIEW}

Being deemed as a standard data acquisition and instrument control software, LabVIEW is not only an innovative software product launched by National Instruments, but also is an integrated environment concerning graphical software development which is most widely used, develops fastest, and has the strongest function now[5]. With the application of graphical programming mode based on flow chart, LabVIEW is a software development environment with a complete function as well as a programming language with a powerful function[6-7].

The software of power quality monitoring system is divided into six functional modules which are data acquisition, data processing, data display, data storage, data analysis and data report, to achieve the acquisition, processing, display, storage and analysis of data with the general process as below:

The system makes data acquisition towards some panel points every $500 \mathrm{~ms}$, and samples each panel point for two periods, and then, makes data processing for the first time to the digital signals collected;

According to the state-level standards concerning power quality in China, the system makes data processing for the second time to the said digital signals every $3 \mathrm{~s}$ to gain the measured values, and then, detects whether or not the measured values are out-of-limit, meanwhile, presents the real-time display for the measured values and the running status of the panel points;

Through an access to the database using LabSQL, set the time of 30 s to store the information of harmonic and the time of $5 \mathrm{~min}$ to store the information of some power quality parameters such as voltage and current, etc.

Through an access to the database using LabSQL, analyze and display the historical data;

Through an access to the database using LabSQL, generate the power quality report for a certain period of time.

\section{A. Data acquisition}

Sequential structure is utilized in the functional module of data acquisition, and DAQmx VI is applied, the signal type is voltage (The current signal has been transformed into voltage signal since resistances are added on the two line sides of the current transformer.), the three-phase voltage and current signals of some panel points are collected for three times (with 3, 3 and 1 respectively as the number of panel points) with the sampling frequency of $12800 \mathrm{~Hz}$ and the number of samples of 512 points, and the collecting principle is as shown in Figure 2.

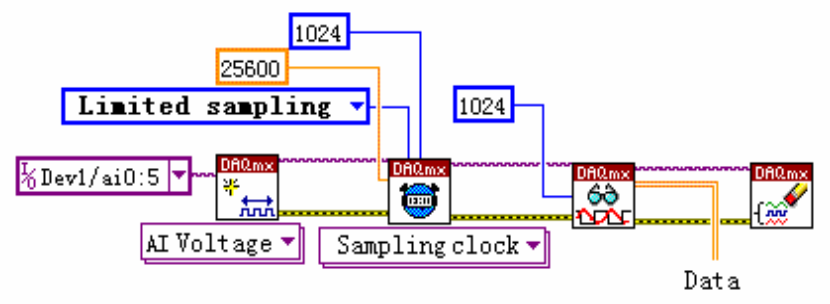

Figure 2. The collecting principle

\section{B. Data processing and display}

The voltage and current signals collected need two times of data processing. Data processing for the first time should be made after collecting signals at each panel point, and, $3 \mathrm{~s}$ later, data processing for the second time should be made, then, enter the judgment sub-procedure to conduct the judgment. The voltage and current signals collected need two times of data processing, this is because that, the measured effective values of the voltage and current, active power, reactive power, power factor and frequency are all instantaneous values; the unbalance of the three-phase voltage is determined according to the R.M.S of 3s.; the amplitude value of harmonic of the three-phase voltage and current at 50th fundamental wave is the average value of the measured values in $3 \mathrm{~s}$, and the total harmonic distortion, odd harmonic distortion and even harmonic distortion are all calculated through the measured value of the amplitude of harmonic of the three-phase voltage and current at 50th fundamental wave; and the fluctuation ratio of the threephase voltage is calculated through taking the voltage value in a certain time. Therefore, data processing for the first time is made after collecting signals at each panel point, and the measured effective values of the voltage and current, active power, reactive power, power factor, frequency, unbalance of the three-phase voltage, fluctuation ratio of the threephase voltage, and the total harmonic distortion, odd harmonic distortion, even harmonic distortion, and instantaneous value of harmonic amplitude at 50th fundamental wave of the three-phase voltage and current; then, time $3 \mathrm{~s}$ to make data processing for the second time to gain the unbalance of the three-phase voltage, the amplitude value of harmonic at 50th fundamental wave, total harmonic distortion, odd harmonic distortion, and even harmonic distortion of the three-phase voltage and current, and then, in combination with the measured effective values of the voltage and current, active power, reactive power, power factor, and frequency in the data processing for the first time to construct a two-dimensional array to enter the judgment sub-procedure for judgment, and then, display the measured value, the running status of the instrument and the warning message on the front panel, as shown in Figure3 . 


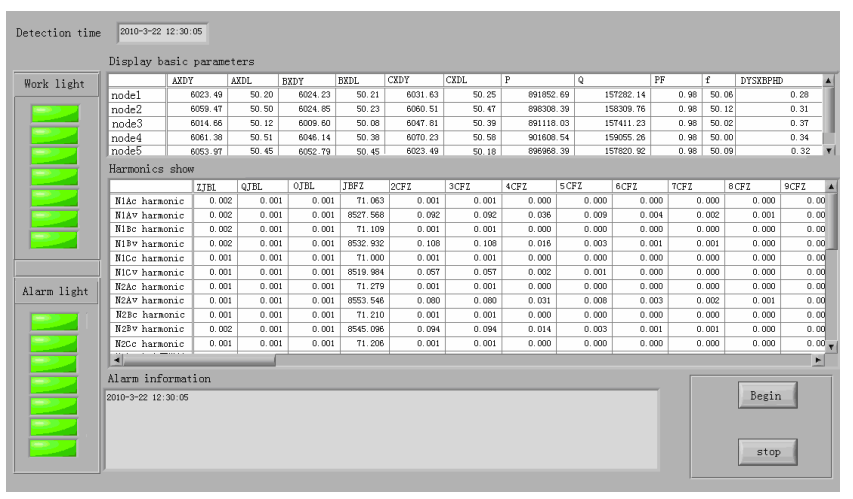

Figure 3. Display the running status

\section{Data storage, analysis and repor}

Making an access to the database using LabSQL, the three functional modules - data storage, analysis and report achieve storage and analysis of the data and generate the report. LabSQL is a multi-database and cross-platform access tool pack for LabVIEW database, supports any database based on OBDC in Windows(Access, SQL,

Server, Oracle, Sybase, and so on), uses Microsoft ADO and SQL language to complete the access to the database, executes SQL query, and operates the record. Before applying LabSQL, a DSN (Data Source Name), based on which the connection between LabSQL and database is set up, should be established in the data source of ODBC(Open Data base Connectivity) in Windows.

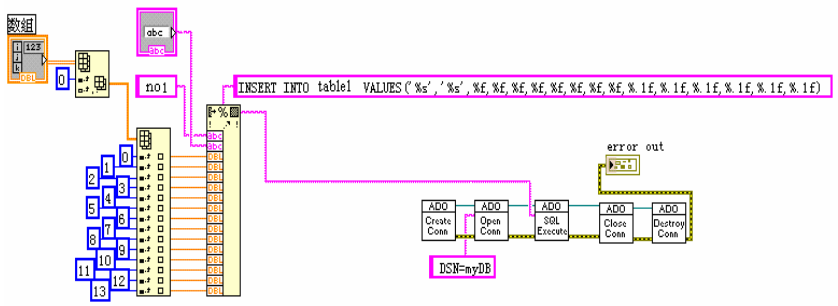

Figure 4. The storage principle

Data storage is divided into two parts - harmonic storage and the storage of other power quality parameters, and is performed according to the setting time interval. It is convenient for the storage and analysis of data to store the measured value in Microsoft Access database. In order to avoid the competition between harmonic and other power quality parameters when storing them at the same time, so in the storage of a same second, harmonic should be stored earlier than the other power quality parameters. In data storage, with the statement of INSERT, insert a line of records into an appointed table, such as INSERT INTO table1 * VALUES node1, 2010-05-10 09: 10: 10, $6023.49,50.20,6024.23,50.21,6031.63,50.25$, $891852.69,157282.14,0.98,50.06,0.28$. The storage principle is as shown in Figure4.

The data analysis and data report modules realize their access to the database using LabSQL, and apply SQL query command-SELECT to gain the records needed from the database table, such as SELECT harmonic FROM table2 WHERE time BETWEEN 2010-05-10 09:10:10 AND 201005-10 09:40:10 ORDER BY time ASC. Data analysis chooses the data demanded to be displayed by operating the select button, such as voltage, and current, etc. and then, figures out the maximum, minimum and average values of these data, and uses an oscillogram to show the data waveform, to make an analysis and display of the data. Data report reads the corresponding data through access and generates the power quality report (HTML doc. and RTF doc.) of a certain period of time. Data analysis is as shown in Figure 5
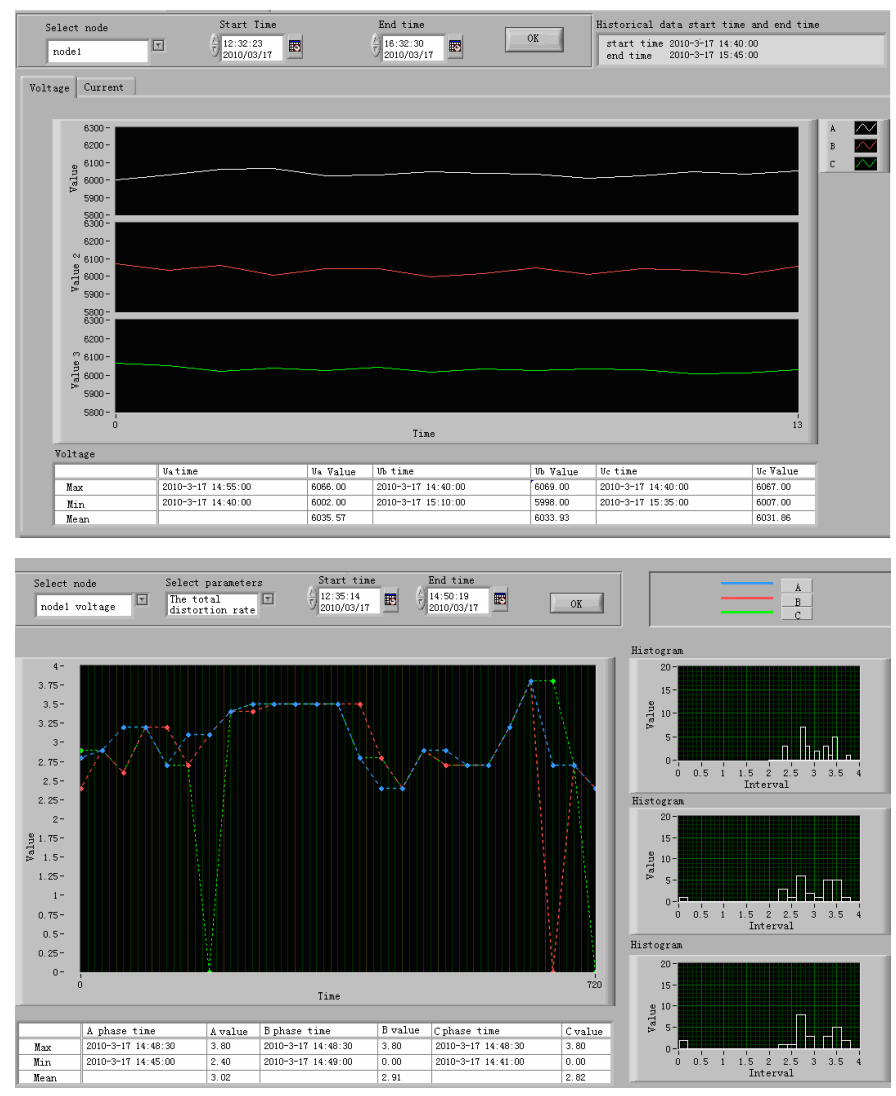

Figure 5. Data analysis

\section{CONCLUSIONS}

The power quality monitoring system introduced in the thesis uses LabVIEW as the development platform, applies LabSQL to realize an access to Microsoft Access database, achieves data acquisition, processing, storage and analysis of the power quality parameters from the panel points selected, is characterized by a low cost, powerful function, easy installation and being widely used, etc. and has a fairly nice use value for the power quality monitoring and analysis concerning factories.

\section{ACKNOWLEDGMENT}

The research was Supported by National Science and Technology Foundation ofChina (2007BAE17B03). 


\section{REFERENCES}

[1] Dong Qiguo. Power Quality Technical Q \& A. Beijing: China Electric Power Press[M], 2003.

[2] Xiao Xiangning. Power Quality Analysis and Control, Beijing: China Electric Power Press[M], 2004.

[3] Shi Yiping; Wu Guo-an. Power Quality Analyzer Based on DSP, Electrical Measurement \& Instrumentation[J], 2002,39, PP17-19.

[4] Wang Bin; Pan Zhencun. Data Synchronous Processing and Device on the Monitoring of Electric Power Quality, Automation of Electric Power Systems[J], 2002,26, PP45-49.
[5] Zhang Tong; Chen Guoshun; Wang Zhenglin. Proficient in LabVIEW programming, Beijing: Electronic Industry Press[M], 2008.

[6] Yang Leping; Li Haitao; Zhao Yong. LabVIEW Advanced Programming, Beijing : Tsinghua University Press[M], 2003.

[7] Jeffrey Traivis; Jim Kring. LabVIEW for Everyone, Beijing: Publishing House of Electronics Industry[M], 2008. 